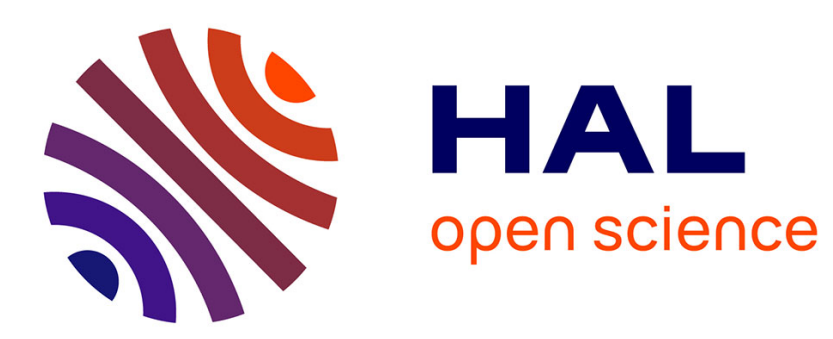

\title{
Single- and multi-objective genetic algorithm optimization for identifying soil parameters
}

Aurélie Papon, Yvon Riou, Christophe Dano, Pierre-Yves Hicher

\section{To cite this version:}

Aurélie Papon, Yvon Riou, Christophe Dano, Pierre-Yves Hicher. Single- and multi-objective genetic algorithm optimization for identifying soil parameters. International Journal for Numerical and Analytical Methods in Geomechanics, 2012, 36 (5), pp.597-618. 10.1002/nag.1019 . hal-01007307

\section{HAL Id: hal-01007307 https://hal.science/hal-01007307}

Submitted on 18 Apr 2017

HAL is a multi-disciplinary open access archive for the deposit and dissemination of scientific research documents, whether they are published or not. The documents may come from teaching and research institutions in France or abroad, or from public or private research centers.
L'archive ouverte pluridisciplinaire HAL, est destinée au dépôt et à la diffusion de documents scientifiques de niveau recherche, publiés ou non, émanant des établissements d'enseignement et de recherche français ou étrangers, des laboratoires publics ou privés. 


\title{
Single- and multi-objective genetic algorithm optimization for identifying soil parameters
}

\author{
A. Papon, Y. Riou, C. Dano and P.-Y. Hicher \\ Research Institute in Civil and Mechanical Engineering, UMR CNRS 6183 - Ecole Centrale Nantes, \\ University of Nantes, 1 rue de la Nö̈, BP 92101, 44321 Nantes cedex 3, France
}

This paper discusses the quality of the procedure employed in identifying soil parameters by inverse analysis. This procedure includes a FEM-simulation for which two constitutive models - a linear elastic perfectly plastic Mohr-Coulomb model and a strain-hardening elasto-plastic model—are successively considered. Two kinds of optimization algorithms have been used: a deterministic simplex method and a stochastic genetic method. The soil data come from the results of two pressuremeter tests, complemented by triaxial and resonant column testing. First, the inverse analysis has been performed separately on each pressuremeter test. The genetic method presents the advantage of providing a collection of satisfactory solutions, among which a geotechnical engineer has to choose the optimal one based on his scientific background and/or additional analyses based on further experimental test results. This advantage is enhanced when all the constitutive parameters sensitive to the considered problem have to be identified without restrictions in the search space. Second, the experimental values of the two pressuremeter tests have been processed simultaneously, so that the inverse analysis becomes a multi-objective optimization problem. The genetic method allows the user to choose the most suitable parameter set according to the Pareto frontier and to guarantee the coherence between the tests. The sets of optimized parameters obtained from inverse analyses are then used to calculate the response of a spread footing, which is part of a predictive benchmark. The numerical results with respect to both the constitutive models and the inverse analysis procedure are discussed.

KEY WORDS: soil parameter identification; inverse analysis; genetic algorithms; multi-objective algorithm

\section{INTRODUCTION}

Using constitutive models to design structures with FEM codes requires the identification of a set of soil parameters. Analytical methods adapted to different constitutive models have been developed [1-4]. The further development of more sophisticated constitutive models requires finer and more complex parameter identification processes, partly because the increasing number of parameters cannot be determined from stress-strain curves directly. In this paper, the authors propose a methodology for identifying soil parameters based on inverse analysis. The aim is to determine the unknown values of the constitutive parameters by minimizing the difference between experimental data and predictions of analytical or numerical calculations. Inverse analysis has been successfully developed in the geotechnical area, partly because it produces a relatively objective determination of the parameters, even those that express no physical meaning, and this occurs from any testing procedure and for any constitutive model, provided that it is possible to run an analytical 
or numerical simulation of the soil response [5-7]. The nature of the simulation-analytical or numerical-depends mainly on the complexity of both the constitutive model and the boundary conditions. In this study, the simulation is carried out numerically.

Inverse analysis is generally an ill-posed mathematical problem, for which neither existence nor uniqueness of a solution can be guaranteed. Therefore, a specific approach has to be developed in order to reduce the number of parameters to be identified and to restrict the parameter search space, through an initial sensitivity study and a priori knowledge about the behavior of the studied soil. However, even with this preliminary study, the problem of non-uniqueness remains an open question. Indeed, inherent experimental and numerical uncertainties and the imperfect reproduction of the soil behavior by constitutive models have to be considered: a usual way is to accept a certain error-which has to be defined for each inverse analysis process- to define the best sets of parameters as developed by Levasseur et al. [8-10]. For these theoretical and practical reasons, satisfactory parameter identification needs not to be extremely precise, but must be reliable. Hence, determining a set of satisfactory solutions is preferable to the search of a unique precise solution.

Given the fact that tests are rarely conducted solely, it stands to reason to process all the experimental data simultaneously. Tests conditions could generate different sets of optimized parameters if the inverse analysis is executed separately on each test. To avoid indecision and indetermination, an alternative way to predict a satisfactory set of parameters is to involve simultaneously many experimental responses through the same inverse analysis.

The aim of this paper is to present a parameter identification methodology which can determine a set of satisfactory solutions for different tests. First, the inverse analysis is formulated as an optimization problem and different resolution methods are presented and compared. Then, parameter identification from two pressuremeter tests is carried out by considering successively two different constitutive models. The validity of the method is discussed by confronting the numerical results of spread footing settlement to the experimental ones obtained from an international benchmark [11]. Finally, conclusions are made about which optimization method is best to select.

\section{FORMULATION AND RESOLUTION OF INVERSE ANALYSIS}

Inverse analysis is based on the formulation of an optimization problem. However, it is important to distinguish the resolution of a mathematical optimization and the corresponding parameter identification. In this section, we focus on the mathematical problem and the ability of the resolution methods to approach and detect optima. In the following sections, we consider both mathematical and geotechnical standpoints.

\subsection{Formulation of a classical inverse analysis problem}

In order to carry out an inverse analysis, the user has to formulate first the optimization problem, which means to define the function to be minimized, called error or cost function, and the search space. Error functions are currently given by

$$
F_{\text {err }}(x)=\left[\sum_{i=1}^{N}\left|d_{\text {exp }}^{i}-d_{\text {num }}^{i}(x)\right|^{k}\right]^{1 / k}
$$

where $x$ is a vector of parameters, $d_{\exp }^{i}$ the experimental value at the measurement point $i$ and $d_{\text {num }}^{i}$ the computed value, $k$ is a non-null positive real and $N$ is the number of values.

According to Tarantola [12], the least-squares criterion $(k=2)$ is highly sensitive to a small number of large errors in a data set. In fact, the higher the parameter $k$, the higher the sensitivity to errors in a data set. If in situ experimental results are involved in inverse analysis as in this study, scattered data should be expected and $k$ should be considered equal to 1 . Moreover, in order to make the error function independent of the interval between the measurement points, this function is defined as the area between the experimental and the computational curves, i.e. the integral of 


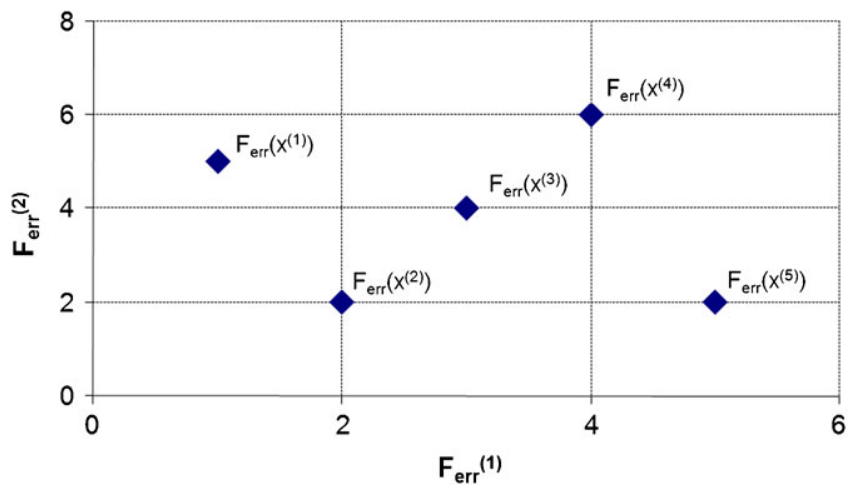

Figure 1. Solutions of a two-objective optimization.

the absolute value of the error during the test. A justification of this definition in the particular case of this study is given in Section 3.2.4.

The search space for the vector $x$ has to be chosen by considering all the physically acceptable values for each parameter. In order to minimize the problem of non-uniqueness in the solution, some initially undetermined parameters can also be fixed, either because a preliminary sensitivity analysis has shown that they have no effect on the predicted response, or because some additional information allows the user to determine their values.

\subsection{Multi-objective analysis}

Contrary to the current approach which considers inverse analysis as a single-objective problem, using only one test result and one type of simulation, a multi-objective problem aims at coupling multiple test results and simulations. Such a formulation results in multiple error functions.

In the case of single-objective problems, the comparison between two sets of parameters is obvious, in so far as the lower the value of the error function, the better the corresponding set of parameters. In the case of multi-objective problems, the comparison is not so easy anymore, if the user does not make any preference among the objectives. For example, five sets of parameters and the corresponding values of the error functions $F_{\text {err }}^{(1)}$ and $F_{\text {err }}^{(2)}$ are considered in a two-objective problem in Figure 1. The set of parameters $x^{(1)}$ fits better the first experimental curve than the set of parameters $x^{(2)}$, whereas inversely the set of parameters $x^{(2)}$ fits better the second experimental curve than the set of parameters $x^{(1)}$. Without any a priori preference, we cannot conclude whether $x^{(1)}$ is better than $x^{(2)}$ or vice versa.

Different resolution approaches have been developed to solve a multi-objective problem. Deb [13] distinguishes the approaches that require making preferences before optimization (a priori approaches) and the ones which require making preferences after optimization (a posteriori approaches). An intuitive a priori approach, called the weighted sum approach, consists of building a global error function from the initial error functions, which are weighted considering a priori preferences. For geotechnical problems, it means that the user is able to decide a priori which test is more reliable than another. All the a posteriori approaches are aimed at determining the so-called Pareto frontier. Originally introduced by Pareto [14], the Pareto frontier is used in order to help decision making in the optimization problem. By definition, a solution $x^{(i)}$ dominates a solution $x^{(j)}$ in terms of Pareto, if both conditions of Equation (2) are fulfilled.

$$
\begin{aligned}
& \forall m \in[1 ; M], F_{\text {err }}^{(m)}\left(x^{(i)}\right) \leqslant F_{\text {err }}^{(m)}\left(x^{(j)}\right) \\
& \exists m \in[1 ; M], F_{\text {err }}^{(m)}\left(x^{(i)}\right)<F_{\text {err }}^{(m)}\left(x^{(j)}\right)
\end{aligned}
$$

where $M$ is the number of test results.

In the example given in Figure $1, x^{(1)}$ dominates $x^{(4)}$, and $x^{(2)}$ dominates $x^{(3)}, x^{(4)}$ and $x^{(5)}$. Likewise, $x^{(3)}$ and $x^{(5)}$ are not comparable in terms of Pareto. The solutions of a multi-objective 
problem correspond to the Pareto optimal solutions, i.e. the solutions which are not dominated by any other sets of parameters. In the previous example, $x^{(1)}$ and $x^{(2)}$ are equivalent solutions of the two-objective problem and $F_{\mathrm{err}}\left(x^{(1)}\right)=\left(F_{\mathrm{err}}^{(1)}\left(x^{(1)}\right), F_{\mathrm{err}}^{(2)}\left(x^{(1)}\right)\right)$ and $F_{\mathrm{err}}\left(x^{(2)}\right)=$ $\left(F_{\text {err }}^{(1)}\left(x^{(2)}\right), F_{\text {err }}^{(2)}\left(x^{(2)}\right)\right)$ belong to the Pareto frontier. After the optimization with an a posteriori approach, the user can select the most suitable set of parameters based on the knowledge of the Pareto frontier.

\subsection{Optimization algorithms}

2.3.1. Deterministic algorithms. Single-objective inverse problems in the geotechnical area have been commonly carried out by using gradient methods [15-20]. However, the robustness of the simplex method [21], also known as the Nelder and Mead downhill method, has been preferred by Gioda [5] because it does not require the derivative of the error function contrary to the algorithms based on local gradients. A simplex is a polyhedron containing $n+1$ points in a $n$-dimensional space, where $n$ is the number of parameters to be optimized. Under the simplex method, the initial simplex is modified according to the value of the error function at the $n+1$ vertexes, using the following three operations: reflection, contraction and expansion. The calculation is stopped when the improvement of the function error becomes smaller than a given tolerance value.

Both gradient and simplex methods determine a unique set of parameters. However, the uniqueness of the solution is not guaranteed. Zentar et al. [18] highlight the fact that the increasing number of optimized parameters exacerbates that shortcoming. Ledesma et al. [22] and Gens et al. [23] have alternatively proposed a probabilistic approach in order to take experimental uncertainties into account and to limit the problem of non-uniqueness of the solution.

Another weakness of the gradient and simplex methods is that they strongly depend on the initial set of parameters, whenever the error function has several secondary minima. This drawback can be overcome by multiple optimizations starting from different initial sets of parameters, in order to detect convergences toward secondary minima. Hicher and Rahma [24] have developed empirical correlations between the physical properties of soils (e.g. nature of constituent, grain size distribution) and their mechanical properties. These considerations allow the estimation of a realistic initial set of parameters. Obrzud et al. [25] have proposed to proceed in two steps. At first, they use a neural network technique to estimate the parameter values. These values are then optimized by means of a gradient-based algorithm, which improves the quality of the initial set. The method exploits the robustness of the neural network technique and the precision of the gradient methods.

In a multi-objective problem, if the gradient or simplex methods have to be used, the weighted sum approach can be selected. If preferences between the objectives can be made, an a priori approach is suitable. For example Dano et al. [19] have performed an inverse analysis from two dilatometer tests: one in fully drained conditions and another one in partially drained conditions, in order to identify the plastic compressibility, the pre-consolidation pressure and the slope of the critical state line of heavy oil reservoir soils. They gave an important weight to the first pressure-strain curve because of its high reliability compared with the other one. If a priori preferences cannot be justified, the weights associated with each initial error function can be modified as proposed by Mertens et al. [26] in order to determine the Pareto frontier. However, this method does not guarantee that all the solutions can be determined along the Pareto frontier in case of encountering locally non-convex Pareto surfaces. Moreover, if the weights are changed regularly, it does not mean that the Pareto solutions are regularly located on the Pareto surface and, therefore, the Pareto surface has to be determined with most care.

2.3.2. Genetic algorithms. Genetic algorithms $[27,28]$ offer many advantages compared with the previous methods [13]. Genetic algorithms are derived from Darwin's evolution theory. They belong to the family of stochastic algorithms and reproduce biological processes. The probability of survival of the best adapted individuals, represented by the best set of parameters, and the probability of the 
emergence of competitive ones are improved by the transmission of a favorable gene pool. Gene mutation preserves the genetic diversity of the population and makes possible the emergence of new competitive individuals. The solution can be defined as the set of individuals whose values of the error function are inferior to a predetermined value and corresponds to individuals with different gene pools. The result provides a global view of this set of gene pools. The strategy of the algorithm is to detect individuals with low error functions, using a reduced number of iterations compared with an exhaustive search, rather than to guarantee the detection of an optimal set of parameters. First, an initial population is randomly generated among the search space. Then, at each generation, this population is modified according to a pseudo-random process based on the value of the error function through the following operations: selection, cross-over and mutation. Selection and crossover mainly improve the performance of individuals, whereas mutation makes it possible to continue the exploration of a given search domain and to avoid the premature convergence toward a secondary minimum.

Genetic algorithms work with a population of solutions, so that they can provide a set of satisfactory solutions. They also do not use any gradient information and they are based on stochastic principles. Therefore, they can be implemented for different types of problem $[7-10,29,30]$ and are considered more robust than the gradient methods. Moreover, Levasseur et al. $[9,10]$ propose to characterize mathematically the obtained set of satisfactory solutions by a principle component analysis which gives a first-order approximation of the solution as an ellipsoid. When the solution set is not too curved in the research space, the user disposes of an ellipsoid characteristic of the soil properties. The computational cost with genetic algorithms is higher than with gradient methods. However, the trials with different initial sets of parameters for the simplex method increase consequently the computational cost.

Genetic algorithms present also advantages in multi-objective inverse problems, because they can determine reliably the Pareto frontier in one single simulation run [13]. Several multi-objective evolutionary algorithms classified as non-elitist or elitist [13] can be found in the literature. The first ones have been developed earlier and set the principles of a multi-objective evolutionary algorithm. The second ones are supposedly faster and better than the former ones, because of the use of an elite-preserving operator. MOGA-II [31] is one of them and we have subsequently selected it. It is an improved version of Multi-Objective Genetic Algorithm (MOGA), which has been introduced by Poloni and Pediroda [32]. Their study helped to modify the classical single-objective genetic algorithm by introducing first a method of selection based on dominance in terms of Pareto, and then, a new operator called directional cross-over, which aims to determine a direction of improvement by comparing the value of the error function of two individuals. This new operator makes for a faster convergence, but has to be used sparingly for highly non-linear problems in order to avoid local Pareto frontiers. In this study, we do not expect a highly non-linear problem and the computation cost for the evaluation of individuals can be problematic. Therefore, the directional cross-over operator is used with the classical cross-over. An improved Multi-Objective Genetic Algorithm (MOGA-II) is presented by Poles et al. [31]. In MOGA-II, an elite-preserving operator is introduced.

A comparison between the gradient method and genetic algorithms has already been proposed by Levasseur et al. [8] and Rechea et al. [33]. They show that the use of a genetic algorithm to identify soil parameters is particularly suitable when the topology of the error function is complex. We propose to extend this comparison by using two different constitutive models, by introducing multi-objective optimization in geotechnical area and finally by using the simulation of a spread footing test to estimate the quality of the parameter identification.

With respect to the previous conclusions, a comparison between:

- the simplex method and genetic algorithms in case of single-objective problems, on the one hand,

- the weighted sum approach with the simplex method and a multi-objective genetic algorithm with an elite-preserving operator, on the other hand, is performed.

The aim of this study is to establish the relevance of each of them for determining material parameters in geotechnical analysis. 


\section{IDENTIFICATION OF DESIGN PARAMETERS FROM PRESSUREMETER TESTS FOR A SUBSEQUENT PREDICTION OF SETTLEMENTS}

\subsection{Presentation of the benchmark}

As part of a spread footings benchmark [11], five loading tests on square footings ranging from $1 \times 1 \mathrm{~m}$ to $3 \times 3 \mathrm{~m}$ in size were conducted at a sandy site, where numerous soil tests were also performed. The participants of the benchmark had to predict the load corresponding to settlements of 25 and $150 \mathrm{~mm}$ for each of the five footings.

The parameters necessary for the numerical estimation of the foundation settlements will be identified using inverse analysis, as previously mentioned. Only the $3 \times 3 \mathrm{~m}$ square footing test is studied here. Figure 2 shows the general soil layering at the site (see [11] for more information). Two pressuremeter tests have been performed at two different depths: 2 and $5.9 \mathrm{~m}$. Triaxial tests have been performed at three different effective confining pressures for six specimens sampled at 0.6 and $3 \mathrm{~m}$, respectively. According to Briaud and Gibbens [11], the sand is probably lightly overconsolidated by desiccation of fines and removal of about $1 \mathrm{~m}$ of overburden at the location of the spread footing tests, which is confirmed by the pressuremeter tests. The coefficient of earth pressure at rest $K_{0}$ is taken equal to 0.7 , consistently with pressuremeter test results.

\subsection{Pressuremeter tests and numerical modeling}

3.2.1. Pressuremeter curves. In this study, we consider as the basic information on the soil behavior, the pressuremeter curve which gives the evolution of the pressure applied within the probe as function of the ratio $u(a) / a$, where $a$ is the radius of the probe and $u(a)$ is the displacement of the wall. The first part of the experimental curves (up to around $u(a) / a=4 \%$ ) is not taken into account in the calculation of the error function because of the unusual curvature at the beginning of the pressuremeter tests, probably due to the remolding of the soil along the cavity wall [34] (see also Figure 3).

3.2.2. FEM modeling. Owing to the axisymmetry of the problem, a 2D finite element model is sufficient. Moreover, plain-strain condition is assumed in the vertical direction. So, a horizontal layer is constructed in the CESAR-LCPC FEM code to simulate the pressuremeter test. 8-node rectangular elements are used so that the model contains 891 nodes and 252 elements. The ratio of the outer diameter to the inner diameter of the model is taken sufficiently high in order to model the condition of infinite medium [35]. The calculations consider the hypothesis of small strains, which is justified by the values of the local strains which are always smaller than $10 \%$ (without considering the close surrounding area of the probe). The loading is displacement controlled and, at each step, the same displacement increment is applied all along the probe.

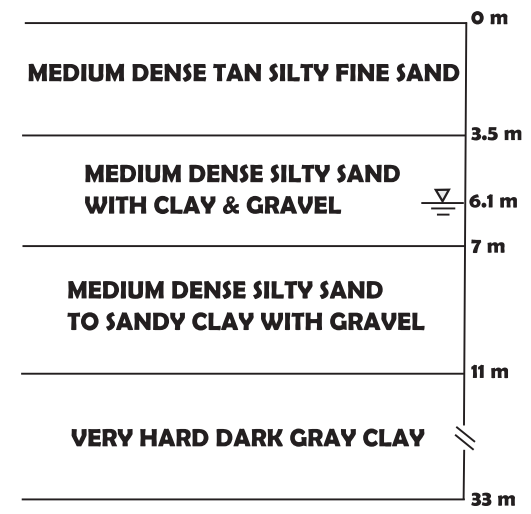

Figure 2. Soil layering (from experimental results [11]). 


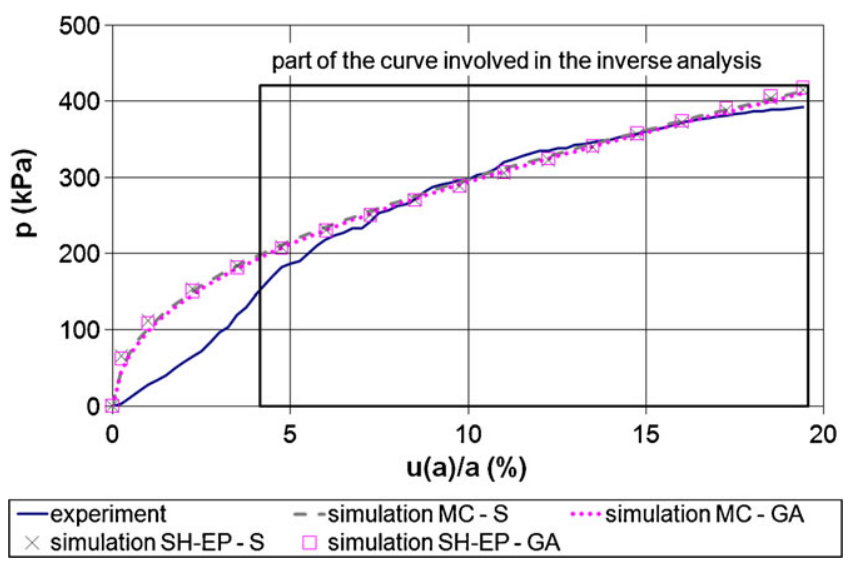

Figure 3. Comparison of the experimental and numerical results of the first pressuremeter test with the simplex (S) and genetic (GA) methods assuming two constitutive models, Mohr-Coulomb model (MC) and a strain-hardening elasto-plastic model (SH-EP).

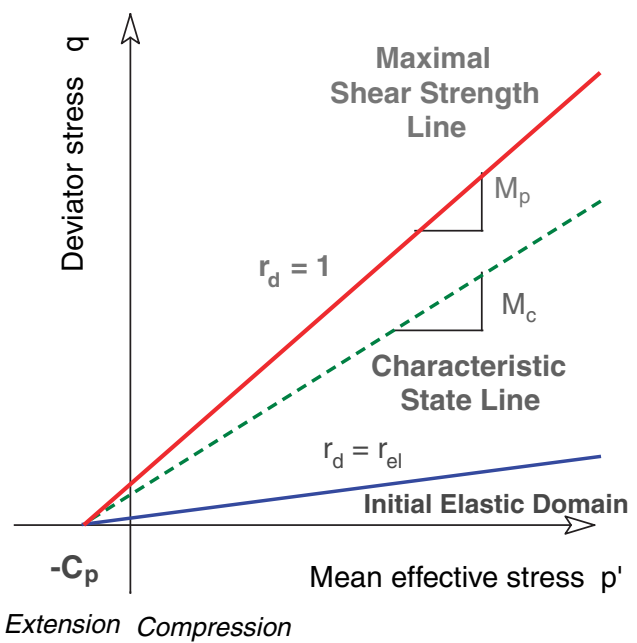

Figure 4. Shape of the strain-hardening yield surface in the $p^{\prime}-q$ plane.

3.2.3. Constitutive models. Because of its relatively extensive use in geotechnical design, the linear elastic perfectly plastic Mohr-Coulomb model is selected. This model is characterized by five parameters: elastic modulus $E$, Poisson's ratio $v$, friction angle $\varphi$, dilatancy angle $\psi$ and cohesion $c$. The identification is performed on the following three parameters: elastic modulus $E$, friction angle $\varphi$ and cohesion $c$, because of their major influence on the design of spread footings. In all the calculations, Poisson's ratio is taken equal to 0.33 and the following correlation between the friction angle and the dilatancy angle, used by many authors, is considered:

$$
\psi=\varphi-30 \text { (degrees) }
$$

However, this model can be seen as a rough estimation of the behavior of a cohesive-frictional soil. Particularly, the non-linear behavior before failure cannot be represented. To circumvent that shortcoming, a strain-hardening elasto-plastic model [36] has been implemented in CESAR-LCPC.

Using a 3D expression of the Mohr-Coulomb yield surface [37], a hardening function $r_{\mathrm{d}}$ is introduced (see also Figure 4):

$$
f\left(p^{\prime}, q, \theta, r_{\mathrm{d}}\right)=\frac{q \cdot m(\theta)}{M_{p}}-\left(p^{\prime}+C_{p}\right) \cdot r_{\mathrm{d}}\left(\varepsilon_{\mathrm{d}}^{\mathrm{p}}\right)=0
$$




$$
m(\theta)=\frac{6}{\sqrt{3}\left(3-\sin \varphi_{p}\right)}\left[\cos \theta-\frac{\sin \varphi_{p}}{\sqrt{3}} \cdot \sin \theta\right]
$$

where $\theta$ is Lode's angle, $r_{\mathrm{d}}$ is the hardening function depending on the deviatoric plastic strain $\varepsilon_{\mathrm{d}}^{\mathrm{p}}$, $M_{p}$ is the slope of the maximum shear strength line in the $p^{\prime}-q$ plane, which can also be deduced from the more commonly used friction angle $\varphi_{p}$ through Equation (6) and $C_{p}$ is the isotropic tensile strength which is related to the Mohr-Coulomb cohesion $c$ through Equation (7):

$$
\begin{aligned}
\varphi_{p} & =\operatorname{Arcsin}\left(\frac{3 \cdot M_{p}}{6+M_{p}}\right) \\
C_{p} & =\frac{c}{\tan \varphi_{p}}
\end{aligned}
$$

The non-linear behavior is described assuming a hyperbolic hardening function:

$$
r_{\mathrm{d}}\left(\varepsilon_{\mathrm{d}}^{\mathrm{p}}\right)=r_{\mathrm{el}}+\frac{\varepsilon_{\mathrm{d}}^{\mathrm{p}} \cdot\left(1-r_{\mathrm{el}}\right)}{a+\varepsilon_{\mathrm{d}}^{\mathrm{p}}}
$$

where $r_{\mathrm{el}}$ defines the size of the initial elastic domain (when $\varepsilon_{d}^{\mathrm{p}}=0$ ). The parameter $a$ characterizes the hardening rate: an increase in the value of $a$ involves an apparent more compressible volumetric response and an apparent more ductile behavior. In fact, the maximum dilation and the maximum strength are only shifted at greater axial strains. Therefore, a greater value of $a$ increases the non-linearity of the stress-strain response, whereas a very low value of $a$ renders the model similar to the elastic perfectly plastic model.

The calculation of plastic strains is based on the following flow rule:

$$
\frac{\mathrm{d} \varepsilon_{\mathrm{v}}^{\mathrm{p}}}{\mathrm{d} \varepsilon_{\mathrm{d}}^{\mathrm{p}}}=\left[\frac{\sqrt{3} \cdot\left(3-\sin \varphi_{c}\right)}{6 \cdot\left(\cos \theta-\frac{\sin \varphi_{c}}{\sqrt{3}} \cdot \sin \theta\right)} \cdot M_{c}\right]-\frac{q}{p+C_{p}}
$$

where $\mathrm{d} \varepsilon_{\mathrm{v}}^{\mathrm{p}}$ and $\mathrm{d} \varepsilon_{\mathrm{d}}^{\mathrm{p}}$ are the volumetric plastic and deviatoric plastic strain increments, respectively. $M_{c}$ is the slope of the line representing the transitional state between the contractant and dilatant domains (usually called characteristic state or phase transformation state) in the $p^{\prime}-q$ plane $\left(\varphi_{c}\right.$ is the characteristic angle corresponding to $M_{c}$ ).

The dependency of the small strain stiffness on the mean effective stress has also been introduced in the strain-hardening elasto-plastic model in order to take into account the evolution of elastic properties with the effective stresses. A simple formulation in which Poisson's ratio is kept constant has been chosen

$$
\frac{E}{E_{\text {ref }}}=\left(\frac{p^{\prime}}{p_{\text {ref }}^{\prime}}\right)^{n}
$$

where $p_{\text {ref }}^{\prime}, n$ and $E_{\text {ref }}$ are parameters to be experimentally calibrated. The value of $n$ must be adjusted to make it consistent with the strain level at which elastic properties are considered. More precisely, small strain stiffness determined at strain levels below $0.001 \%$ is usually associated with values of $n$ close to 0.5 , whereas the use of a secant modulus identified at a strain level of about $1 \%$ is related to a value of $n$ close to $1[38,39]$. On the conceptual basis of the strain-hardening elasto-plastic model, a value of $n$ equal to 0.5 is a priori considered.

The strain-hardening elasto-plastic model is characterized by seven parameters: reference elastic modulus $E_{\text {ref, }}$ Poisson's ratio $v$, characteristic angle $\varphi_{c}$, friction angle $\varphi_{p}$, cohesion $c$, hardening parameter $a$ and the size of the elastic domain $r_{\mathrm{el}}$. The following four parameters: reference elastic modulus $E_{\text {ref }}$ ( $p_{\text {ref }}^{\prime}$ is set equal to $100 \mathrm{kPa}$ ), friction angle $\varphi_{p}$, cohesion $c$ and hardening parameter $a$ have a major influence on the calculation of spread footings. Therefore, high attention has to be paid to their identification. In order to compare the results of the identification procedure with both models, we decided to keep the number of parameters to identify equal to three. Therefore, 
the results of triaxial tests performed on remolded samples are also considered. The remolding of the soil can have a strong influence on the initial part of the stress-strain curve, but we can assume that the failure characteristics remain the same since the triaxial tests were carried out at the estimated in situ water content and the estimated in situ relative density. Therefore, according to the results of the triaxial tests, a friction angle equal to $35^{\circ}$ was retained. In all the calculations, Poisson's ratio $v$ was taken equal to 0.2 , the characteristic angle $\varphi_{c}$ taken equal to $30^{\circ}\left(M_{c}=1.2\right)$, in agreement with the triaxial test results, and the size of the elastic domain $r_{\mathrm{el}}$ taken equal to 0.7, considering that the soil is lightly overconsolidated with $K_{0}=0.7$.

The two constitutive models discussed above are based on a Mohr-Coulomb failure criterion. The strain-hardening elasto-plastic model takes into account two additional phenomena: nonlinear behavior due to plastic hardening and non-linear elasticity. Therefore, we can expect a better reproduction of the soil behavior. For the two models, identification by inverse analysis is limited to three parameters $(E, \varphi, c)$ and $\left(E_{\mathrm{ref}}, a, c\right)$. The other parameters are determined thanks to correlations, classical values and some other tests. Note that the value of the friction angle is set from triaxial tests only for the second model. Indeed, the second model introduces a hardening function, which allows for a better reproduction of the non-linear behavior before failure and therefore leads to a friction angle value close to a physical value in case of inverse analysis. The bilinear behavior given by the Mohr-Coulomb model implies a compromise between the elastic modulus and the friction angle, which does not lead in the inverse analysis to values corresponding systematically to physical values. Considering this prior determination of some of the parameters, the purpose of this study is to test the ability of inverse analyses to determine the complementary part of the parameter set.

3.2.4. Computational program. The identification procedure is based on the successive use of two different codes: CESAR-LCPC for the direct modeling scheme and ModeFrontier [40] for the optimization process. For each optimization, each initial simplex for the simplex method and each population for the genetic algorithms are randomly generated with the algorithm SOBOL [41] which is used to fill uniformly the search space, i.e. the space of the parameters to be identified. In the case of the Mohr-Coulomb model, the ranges of the possible parameter values correspond to classical values (Table I). The low values of cohesion (inferior to $10 \mathrm{kPa}$ ) can be explained by the non-saturation of the sandy soil. In the case of the strain-hardening elasto-plastic model, resonant column test results [11] on remolded samples give the range of values of $E_{\text {ref }}$. Several simulations with different values of $a$ and mean values of $c$ and $E_{\text {ref }}$ were performed in order to locate the range of study for $a$ (Table II). The initial population for single- and multi-objective problems is set to 200 individuals and the size of the population is kept constant during the optimization process. The probability of directional cross-over is set to 0.5 , the probability of selection to 0.05 and the probability of mutation to 0.1 . Therefore, the probability of classical cross-over is set to 0.35. These values follow the recommendations of the user manual [40] and are similar to the

Table I. Search domain for the linear elastic perfectly plastic Mohr-Coulomb model.

\begin{tabular}{lccc}
\hline & Minimal value & Maximal value & Step \\
\hline$E$ (MPa) & 10 & 50 & 1 \\
$\varphi$ (degrees) & 30 & 50 & 1 \\
$c$ (kPa) & 0 & 10 & 0.5 \\
\hline
\end{tabular}

Table II. Search domain for the strain-hardening elasto-plastic model.

\begin{tabular}{lccl}
\hline & Minimal value & Maximal value & Step \\
\hline$E_{\text {ref }}(\mathrm{MPa})$ & 180 & 280 & 5 \\
$a$ & 0.0025 & 0.05 & 0.0025 \\
$c(\mathrm{kPa})$ & 0 & 10 & 0.5 \\
\hline
\end{tabular}


ones given in the literature [42]. For more details about the binary encoding of individuals see References $[31,32,40]$. Optimization is considered as completed when no improvement concerning the determination of the Pareto frontier is made for five consecutive generations. The simplex algorithm stops the procedure when the relative improvement between two consecutive solutions cannot reach beyond $10^{-5}$.

As previously mentioned, the error functions are defined as the area between the experimental and the numerical curves. In the case of a multi-objective problem, the values of several error functions are compared and the definition of the error functions has to be modified in order to render them independent of the experimental curve considered. For this purpose, the area between the experimental and the numerical curves is divided by the studied interval of the cavity deformation. An adimensional error function would have facilitated the comparison between the two pressuremeter tests. But the decision does not influence the results in the case of the multiobjective analysis with genetic algorithms, given the strategy used to determine the Pareto frontier. In the case of the weighted sum approach with the simplex method, it could have an influence. However, since the results for single- and multi-objective methods are in agreement, we may argue that the influence is limited. The selected error function magnifies the impact of the errors on points that correspond to high pressures and high strains, rather than the errors on points which correspond to the beginning of the curve. This strategy is justified by the higher reliability of the measurements at the end of the test, considering the remolding of the soil along the cavity wall at the beginning of the test.

\subsection{Single-objective identification}

In this section, an inverse analysis as a single-objective problem is carried out based on the pressuremeter test performed at $2 \mathrm{~m}$ depth. Additional comments on the inverse analysis of the second pressuremeter test are made. The two constitutive model presented in the previous section are successively examined.

3.3.1. Identification of the elastic perfectly plastic model parameters. In this subsection, we assume a linear elastic perfectly plastic Mohr-Coulomb behavior for the soil and we consider the pressuremeter test at $2 \mathrm{~m}$ depth. As aforementioned, three parameters $(E, c, \varphi)$ have to be determined.

The optimization procedure was first conducted by using the simplex method. Because the results depend on the initial set of parameters, calculations with five different initial simplexes were carried out. Since three parameters are involved in the optimization, an initial simplex consists of four sets of parameters. The results demonstrate the influence exerted by the initial chosen values on the optimized ones. The results of the optimizations, which lead to the final smallest and biggest values of the error function among the trial tests, are summarized in Table III. The scattering toward the value of the error function is important (up to 48\%) and shows the necessary care when carrying out an inverse analysis with the simplex method. According to the principle of the simplex method, we consider as relevant the trial test which leads to the final smallest value of

Table III. Optimization with the simplex algorithm of the 2-m deep pressuremeter test assuming linear elastic perfectly plastic Mohr-Coulomb model.

\begin{tabular}{|c|c|c|c|c|c|c|c|}
\hline \multicolumn{3}{|c|}{ Initial set } & \multicolumn{5}{|c|}{ Optimal set } \\
\hline$E(\mathrm{MPa})$ & $\varphi$ (degrees) & $c(\mathrm{kPa})$ & $E(\mathrm{MPa})$ & $\varphi$ (degrees) & $c(\mathrm{kPa})$ & $F_{\text {err }}^{(1)}(\mathrm{kPa})$ & $\mathrm{Nb}$ of evaluations \\
\hline 38 & 31 & 8.5 & \multirow{4}{*}{50} & \multirow{4}{*}{31} & \multirow{4}{*}{8.5} & \multirow{4}{*}{8.56} & \multirow{4}{*}{20} \\
\hline 27 & 47 & 0.5 & & & & & \\
\hline 48 & 36 & 5.5 & & & & & \\
\hline 29 & 33 & 4.5 & & & & & \\
\hline 43 & 39 & 4.5 & \multirow{4}{*}{16} & \multirow{4}{*}{46} & \multirow{4}{*}{5} & \multirow{4}{*}{12.63} & \multirow{4}{*}{38} \\
\hline 12 & 44 & 7 & & & & & \\
\hline 33 & 33 & 1.5 & & & & & \\
\hline 17 & 41 & 3 & & & & & \\
\hline
\end{tabular}




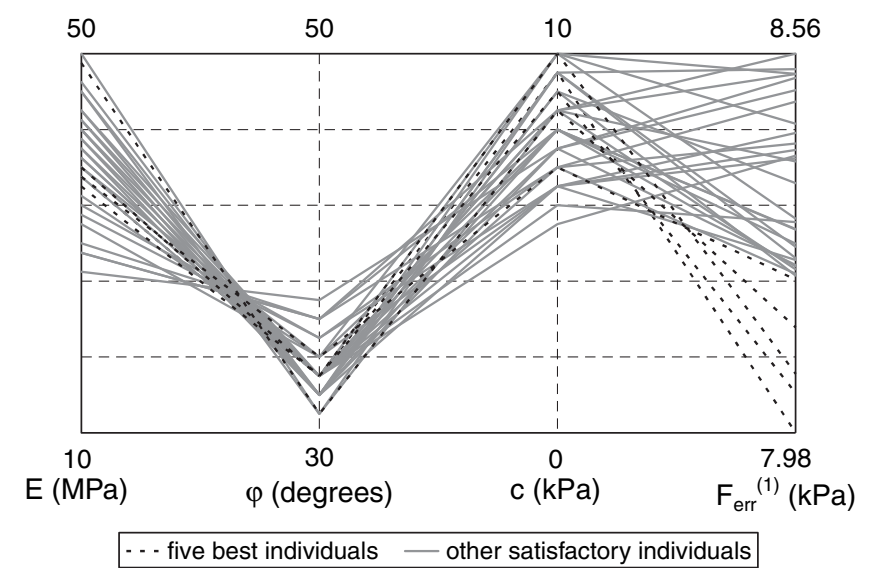

Figure 5. Distribution of the 'satisfactory' individuals obtained with the genetic method assuming Mohr-Coulomb model for the pressuremeter test at $2 \mathrm{~m}$ depth.

Table IV. Five best individuals obtained for the optimization with the genetic method of the 2-m deep pressuremeter test assuming linear elastic perfectly plastic Mohr-Coulomb model.

\begin{tabular}{ccccc}
\hline$E(\mathrm{MPa})$ & $\varphi($ degrees $)$ & $c(\mathrm{kPa})$ & $F_{\text {err }}^{(1)}(\mathrm{kPa})$ & Nb of evaluations \\
\hline 37 & 33 & 9.5 & 7.98 & \\
38 & 33 & 9 & 8.04 & 3000 \\
36 & 33 & 10 & 8.07 & \\
49 & 31 & 8.5 & 8.14 & \\
37 & 34 & 7 & 8.21 & \\
\hline
\end{tabular}

error function. Figure 3 compares the experimental data and the numerical simulation performed with the optimal set of parameters corresponding to the relevant trial test. In this figure, MC stands for the Mohr-Coulomb model. The numerical curve provides an acceptable fitting of the experimental one, if we consider the part of the curve selected for the inverse analysis. However, the value of the friction angle for the optimal set (Table III) is not in agreement with the results of the triaxial tests performed on specimens sampled at $0.6 \mathrm{~m}$ depth (respectively at $3 \mathrm{~m}$ depth), which give a friction angle equal to $34.2^{\circ}$ (respectively $36.4^{\circ}$ ).

Optimization with the genetic method was also carried out. This method provides a population of individuals, which has to be selected according to a satisfaction criterion. All individuals, whose error function is lower than a reference value, are called 'satisfactory'. In this inverse analysis, the genetic method provides a better mathematical optimum than the simplex method. Therefore, the optimum obtained with the simplex method is considered as the reference value. Figure 5 shows the individuals whose error function is lower than the reference value. It is worth noting that for this optimization, the best set of parameters obtained with the simplex method is also detected by the genetic method. About 30 other individuals are more relevant than the mathematical optimum obtained with the simplex method. The parameter values are located in large ranges: for example, the values of the friction angle vary between 31 and $37^{\circ}$. The results are in agreement with the results presented by Levasseur et al. [8], which show a similar distribution of the parameters to be identified. Table IV summarizes the sets of parameters that correspond to the five best individuals. These sets of parameters are included in smaller ranges of values, which indicates a locally smooth error function. The scattering of the satisfactory population in the parameter space (Figure 5) gives qualitative information about the sensitivity of the parameters and their coupling effects. Elastic modulus and friction angle are closely related: an overestimated elastic modulus can be balanced by a smaller friction angle. At a constant friction angle, a connection between the elastic modulus and the cohesion can be established: the higher the elastic modulus, the lower the 
cohesion (see also Table IV). This is in agreement with the fact that the increase in the cohesion or in the friction angle has a similar effect on the computed pressuremeter curve. One way to limit the number of these parameter combinations is to divide the measurements into different sets according to the parameters to be identified. Since the pressuremeter data include information from both elastic and plastic ranges, this procedure would result in two sets of measurements: one for the elastic parameters (beginning of the test) and one for the plastic ones (end of the test). However, in this case, this strategy has to be handled rather carefully because of the influence of the elastic modulus on the final part of the pressuremeter test. Unfortunately, the unreliable measurements probably due to the remolding of the soil at the beginning of the pressuremeter curves prevent us from applying this strategy in the study.

According to the triaxial test results, it seems consistent to select a set of parameters whose friction angle is higher than $33^{\circ}$. Figure 3 compares the experimental data and the numerical simulation performed with the best set of parameters. The numerical curve provides an acceptable matching of the experimental one.

The difference between the numerical curves resulting from the two optimization methods is very small, even if the sets of parameters are quite different (35\% difference in elastic modulus, $6 \%$ difference in friction angle). Note that the numerical curves obtained from the satisfactory sets identified by the genetic algorithm are not represented for the sake of clarity but they are located between the two numerical curves represented in Figure 3. This fact illustrates the non-uniqueness of the solution of the inverse analysis problem and justifies the use of genetic algorithms which guarantee a relatively exhaustive search for satisfactory sets. Moreover, it is worth noting that the genetic algorithm provides better mathematical optimal than the simplex algorithm. It seems that the simplex algorithm remains trapped in a secondary minimum.

Concerning the inverse analysis of the pressuremeter test at $5.9 \mathrm{~m}$ depth, an additional comment can be made. In this case, the simplex method determines a slightly better mathematical optimum than the genetic method (error function: $13.84<13.88 \mathrm{kPa}$ ) if the calculation is stopped when no improvement of the best individual is made for five generations. Thirty-nine generations are necessary for the genetic algorithm to detect the minimum given by the simplex method. This fact illustrates the aim of genetic algorithms which consists of determining a set of satisfactory solutions rather than the exact mathematical one with a pseudo-random search, compared with the aim of the simplex algorithm which consists of finding a mathematical optimum that can be a secondary or a global minimum.

3.3.2. Identification of the strain-hardening elasto-plastic model parameters. In this subsection, we assume a strain-hardening elasto-plastic constitutive model (named SH-EP) for the soil and we consider first the pressuremeter test at $2 \mathrm{~m}$ depth. As aforementioned, three parameters $\left(E_{\text {ref }}, c, a\right)$ have to be determined, $\varphi_{p}$ being set equal to $35^{\circ}$. A process similar to the previous one is followed.

Inverse analyses with the simplex and genetic methods are carried out. Table V summarizes the scattering in the optimal sets, related to the initial set values obtained with the simplex method.

Table V. Optimization with the simplex algorithm of the 2-m deep pressuremeter test assuming a strain-hardening elasto-plastic model.

\begin{tabular}{|c|c|c|c|c|c|c|c|}
\hline \multicolumn{3}{|c|}{ Initial set } & \multicolumn{5}{|c|}{ Optimal set } \\
\hline$E_{\text {ref }}(\mathrm{MPa})$ & $a$ & $c(\mathrm{kPa})$ & $E_{\text {ref }}(\mathrm{MPa})$ & $a$ & $c(\mathrm{kPa})$ & $F_{\mathrm{err}}^{(1)}(\mathrm{kPa})$ & $\mathrm{Nb}$ of evaluations \\
\hline 265 & 0.0225 & 4.5 & \multirow{4}{*}{230} & \multirow{4}{*}{0.045} & \multirow{4}{*}{10} & \multirow{4}{*}{9.05} & \multirow{4}{*}{57} \\
\hline 185 & 0.035 & 7 & & & & & \\
\hline 235 & 0.01 & 1.5 & & & & & \\
\hline 195 & 0.0175 & 5.5 & & & & & \\
\hline 250 & 0.0425 & 0.5 & \multirow{4}{*}{265} & \multirow{4}{*}{0.0225} & \multirow{4}{*}{4} & \multirow{4}{*}{11.01} & \multirow{4}{*}{48} \\
\hline 225 & 0.005 & 8.5 & & & & & \\
\hline 275 & 0.03 & 3 & & & & & \\
\hline 225 & 0.025 & 1.5 & & & & & \\
\hline
\end{tabular}




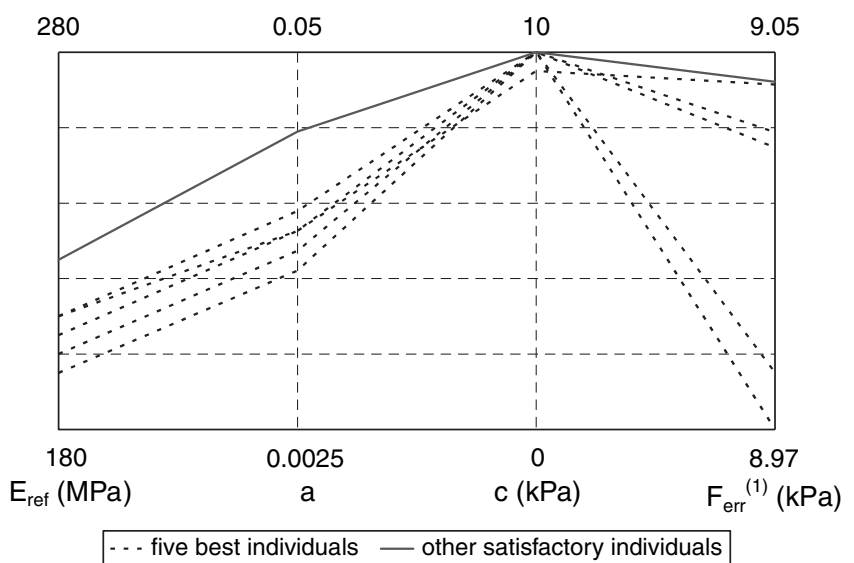

Figure 6. Distribution of the 'satisfactory' individuals obtained with the genetic method assuming a strain-hardening elasto-plastic model for the pressuremeter test at $2 \mathrm{~m}$ depth.

Table VI. Five best individuals obtained for the optimization with the genetic method of the 2-m deep pressuremeter test assuming a strain-hardening elasto-plastic model.

\begin{tabular}{ccccc}
\hline$E_{\text {ref }}(\mathrm{MPa})$ & $a$ & $c(\mathrm{kPa})$ & $F_{\text {err }}^{(1)}(\mathrm{kPa})$ & Nb of evaluations \\
\hline 200 & 0.025 & 10 & 8.97 & \\
205 & 0.0275 & 10 & 8.98 & 1200 \\
195 & 0.0225 & 10 & 9.03 & \\
210 & 0.03 & 10 & 9.03 & \\
210 & 0.0275 & 10 & 9.04 & \\
\hline
\end{tabular}

It is worth noting that the scattering is less important than in the case of the Mohr-Coulomb model, which implies a smoother error function. As previously explained, the lowest value of the error function given by the simplex method is considered as the satisfactory criterion for the genetic method. Figure 6 shows the individuals whose error function is lower than the reference value and Table VI summarizes the five best sets of parameters obtained with the genetic method. Table VI and Figure 6 show a relation between $E_{\text {ref }}$ and $a$-the higher $E_{\text {ref }}$, the higher $a$-and a preference for high values of $c$. These results are in agreement with the comments made in the previous section concerning the Mohr-Coulomb model.

Figure 3 compares the fitting of the experimental curve for the two constitutive models. One can see that the two models produce very similar results. The main difference concerns the beginning of the curve, which is not taken into account in the inverse analysis. The beginning of the curve, unfortunately not usable in this study, is thus crucial to distinguish the quality of the reproduction of the soil behavior by both models.

Considering the satisfactory population obtained from the inverse analysis of the second pressuremeter curve with the genetic method, one can notice that the lower values of cohesion (inferior to $4 \mathrm{kPa}$ ) are obtained. This can be explained by a smaller value of the suction in an area closer to the water table.

This first study compared two kinds of algorithms in the case of single-objective inverse analyses. For both constitutive models, the numerical curves resulting from the inverse analyses with the simplex and genetic methods provide an equivalent fitting of the experimental one, even if the genetic method provides slightly better results if we consider the values of the error function. However, the parameters obtained with the simplex method appear to be inconsistent with the triaxial tests results. The advantage of the genetic method consists of providing a set of satisfactory individuals. From this population, the user gains information about the sensitivity of the numerical response to the parameter values and the possible relations between them. Moreover, considering 
the user's scientific and technical background, and incorporating the results of others tests, the user will be able to select with better accuracy the parameters for the design calculations.

Comparing the identification procedure with the two models can be a difficult task, because just keeping the number of parameters to be identified constant is not enough to assure similar conditions. Indeed, the topology of the error function depends on the constitutive model and its ability to reproduce the reality. However, as soon as the user selects a set of parameters to be identified and keeps fixed values of the other parameters, the error function will change and depend strongly on these choices. In the case of the Mohr-Coulomb model, a large search space is defined without any fixed value or restricted domain for the parameters to be determined. Under these conditions, the uniqueness of the solution is certainly not assured. In the case of the SH-EP model, additional tests are used to fix the value of the friction angle and to restrict the domain of $E_{\mathrm{ref}}$, which could limit the problem of the non-uniqueness of the solution. Figures 5 and 6 confirm these tendencies by showing more satisfactory sets of parameters over a larger range for the Mohr-Coulomb model.

In order to choose the algorithm to be used for the inverse analysis, the user has to take into account two aspects: the expected reliability and wealth of the information, on one hand and the computation cost, on the other hand. Indeed, the richness of the results is gained to the detriment of the calculation time. Considering that five successive calculations were carried out with the simplex, the average calculation cost of the genetic method is about $\rho=9$ times higher than the one of the simplex method. It is worth noting that this ratio $\rho$ is lower $(\rho=6)$ for the SH-EP model than for the Mohr-Coulomb model $(\rho=12)$. Note that these indications are based on several optimizations for which some results are not mentioned in this paper (see Tables III-VI). The difference between the two models can be explained by the topology of the error functions: the evolution of the error function in the case of the Mohr-Coulomb model is not so smooth as in the case of the SH-EP model. These considerations are based on the number of parameter sets evaluated, i.e. the numbers of FE-calculations for each optimization. To complete this comment on the calculation cost, the time related to each evaluation depends on the constitutive model: the more complex the model, the longer the time computation necessary for an evaluation. In our case, the FE-calculation with the Mohr-Coulomb model is six times quicker than with the SH-EP model. The user has to select the constitutive model according to the expected quality of the design calculation.

This comment concerns the present study (identification of three specific parameters from pressuremeter tests) and cannot be easily generalized. Therefore, if the user can assume a priori the topology of the error function considering the constitutive model used and the parameters to be identified, he or she can predict the potential interest of the genetic algorithms which work better with problems with irregular error functions having secondary minima.

At the end of this part of the study, we obtain conclusions similar to the ones presented by Levasseur et al. [8] and Rechea et al. [33]. Furthermore, we highlight the influence of the constitutive models on the topology of the error function.

\subsection{Multi-objective identification}

In the previous section, two separate inverse analyses of the pressuremeter curves were performed. However, the parameters obtained from these two tests may not have been consistent with each other. Such a problem exists especially when we assume a linear elastic perfectly plastic behavior, for which the probability of non-uniqueness of the solution is high. For example, the best sets of parameters obtained with the simplex method give a value of the elastic modulus at $2 \mathrm{~m}$ depth higher $(50 \mathrm{MPa})$ than the elastic modulus at $5.9 \mathrm{~m}$ depth $(36 \mathrm{MPa})$. In the case of the genetic method, the ranges of friction angle values obtained for both depths are so different (inferior to $34^{\circ}$ and superior to $38^{\circ}$ ) that this result does not appear consistent with the relative homogeneity of the soil. By increasing the satisfactory criterion of each pressuremeter test in the case of single-objective optimizations, a set of satisfactory solutions for both tests can be determined. However, this set of solutions is too reduced to be processed and involves high values of the error functions. Indeed, the single-objective optimization explores only localized areas and, therefore, 


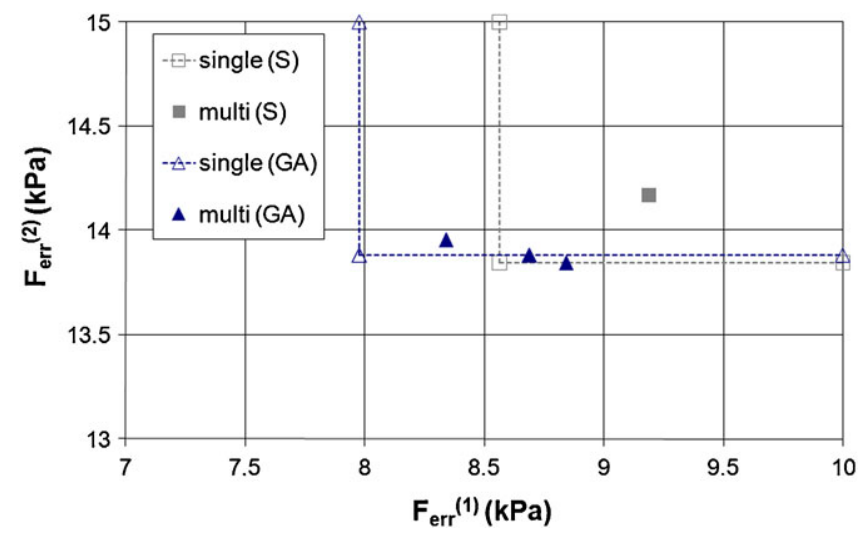

Figure 7. Distribution of Pareto optimal solutions of the multi-objective identification with the simplex (S) and genetic (GA) methods in the criterion space assuming Mohr-Coulomb model compared with the results of single-objective identification.

does not provide enough information outside these areas. Moreover, due to the complexity of some error functions, it appears difficult to extrapolate the information from this reduced set of solutions.

In order to find a compromise which satisfies both tests, and to improve, therefore, the reliability of the results, both pressuremeter tests are simultaneously considered in the inverse analysis, which becomes a multi-objective identification.

3.4.1. Identification with the Mohr-Coulomb model. For the Mohr-Coulomb model, four parameters have to be optimized: two different elastic moduli $E_{1}$ (respectively $E_{2}$ ) which correspond to the two different depths $2 \mathrm{~m}$ (respectively $5.9 \mathrm{~m}$ ), one cohesion $c$ and one friction angle $\varphi$, assuming the homogeneity of the soil profile. It is worth noting that a unique elastic modulus has not been searched for. Therefore, the determination of one elastic modulus is independent from the other and so depends only on the corresponding pressuremeter test. This aspect allows us to circumvent the inability of the Mohr-Coulomb model to represent the evolution of elastic properties with effective stresses.

In the case of the simplex method, the weighted sum approach is applied. The extreme limits of the Pareto frontier have already been given by the single-objective inverse analyses (see Figure 7). As the search for each elastic modulus depends only on the corresponding pressuremeter test, each solution of the single-objective optimization determines the value of one error function. The value of the other error function remains unknown. The dashed lines represent the results of the single-optimization. The symbols are used only to distinguish the two methods (simplex (S) and genetic algorithm (GA)). An additional point is determined for the case where both pressuremeter tests have the same weights. The inverse analysis consists then of minimizing the error function defined by

$$
F_{\mathrm{err}}=\frac{F_{\mathrm{err}}^{(1)}+F_{\mathrm{err}}^{(2)}}{2}
$$

where $F_{\text {err }}^{(1)}$ corresponds to the pressuremeter test at $2 \mathrm{~m}$ depth and $F_{\text {err }}^{(2)}$ to the pressuremeter test at $5.9 \mathrm{~m}$ depth.

Five trial tests with different initial simplexes were performed. The results of the optimizations which lead to the final smallest or biggest values of the error function among the trial tests are summarized in Table VII. The strong dependency of the results on initial simplexes can be explained by the complexity of the error function $F_{\text {err }}$, which superposes the irregularities of the two error functions $F_{\mathrm{err}}^{(1)}$ and $F_{\mathrm{err}}^{(2)}$. The insufficiency of the simplex method is illustrated by the trial 
Table VII. Optimization with the weighted sum approach using simplex method and assuming linear elastic perfectly plastic Mohr-Coulomb model.

\begin{tabular}{|c|c|c|c|c|c|c|c|c|c|c|c|}
\hline \multicolumn{4}{|c|}{ Initial set } & \multicolumn{8}{|c|}{ Optimal set } \\
\hline $\begin{array}{c}E_{1} \\
(\mathrm{MPa})\end{array}$ & $\begin{array}{c}E_{2} \\
(\mathrm{MPa})\end{array}$ & $\begin{array}{c}c \\
(\mathrm{kPa})\end{array}$ & $\begin{array}{c}\varphi \\
\text { (degrees) }\end{array}$ & $\begin{array}{c}E_{1} \\
(\mathrm{MPa})\end{array}$ & $\begin{array}{c}E_{2} \\
(\mathrm{MPa})\end{array}$ & $\begin{array}{c}c \\
(\mathrm{kPa})\end{array}$ & $\begin{array}{c}\varphi \\
\text { (degrees) }\end{array}$ & $\begin{array}{c}F_{\mathrm{err}}^{(1)} \\
(\mathrm{kPa})\end{array}$ & $\begin{array}{c}F_{\text {err }}^{(2)} \\
(\mathrm{kPa})\end{array}$ & $\begin{array}{c}F_{\text {err }} \\
(\mathrm{kPa})\end{array}$ & $\begin{array}{c}\mathrm{Nb} \text { of } \\
\text { evaluations }\end{array}$ \\
\hline 34 & 24 & 8 & 34 & & & & & & & & \\
\hline 24 & 34 & 0 & 50 & & & & & & & & \\
\hline 44 & 13 & 5.5 & 39 & 23 & 37 & 7 & 39 & 9.19 & 14.17 & 11.68 & 62 \\
\hline 16 & 31 & 8.5 & 35 & & & & & & & & \\
\hline 36 & 11 & 3.5 & 46 & & & & & & & & \\
\hline 29 & 29 & 1.5 & 47 & & & & & & & & \\
\hline 49 & 49 & 6.5 & 37 & & & & & & & & \\
\hline 18 & 18 & 4 & 42 & 43 & 42 & 7 & 37 & 89.13 & 17.00 & 53.06 & 58 \\
\hline 29 & 28 & 6 & 41 & & & & & & & & \\
\hline 39 & 33 & 9.5 & 30 & & & & & & & & \\
\hline
\end{tabular}

Table VIII. Pareto optimal solutions obtained for the two-objective optimization with the genetic method assuming linear elastic perfectly plastic Mohr-Coulomb model.

\begin{tabular}{ccccccc}
\hline$E_{1}(\mathrm{MPa})$ & $E_{2}(\mathrm{MPa})$ & $c(\mathrm{kPa})$ & $\varphi($ degrees $)$ & $F_{\text {err }}^{(1)}(\mathrm{kPa})$ & $F_{\text {err }}^{(2)}(\mathrm{kPa})$ & $\mathrm{Nb}$ of evaluations \\
\hline 32 & 37 & 8 & 35 & 8.11 & 86.36 & \\
30 & 41 & 10 & 35 & 8.20 & 53.02 & 4200 \\
23 & 38 & 10 & 38 & 8.34 & 13.95 & \\
21 & 35 & 10 & 39 & 8.69 & 13.88 & \\
22 & 36 & 8.5 & 39 & 8.84 & 13.84 & \\
\hline
\end{tabular}

test, which leads to the worst result $\left(F_{\text {err }}=53.06 \mathrm{kPa}\right)$. If the simplex hits a secondary minimum, it cannot turn back and, therefore, remains trapped.

The use of a multi-objective genetic algorithm (MOGA-II) makes possible a reliable and exhaustive determination of the Pareto frontier in only one calculation run. Five individuals are Pareto optimal solutions. They are summarized in Table VIII. For the sake of clarity, Figure 7 represents a limited objective domain, which contains only three Pareto optimal solutions given by the genetic method. Considering the high values of $F_{\text {err }}^{(2)}$ of the two other Pareto optimal solutions (superior to $50 \mathrm{kPa}$ ), these solutions do not seem suitable for the studied problem and are no longer considered in the following. The results are in agreement with the ones of the single-objective optimization. The multi-objective genetic algorithm detects a solution with a value of $F_{\text {err }}^{(2)}$ lower than the value of $F_{\text {err }}^{(2)}$ corresponding to the best set in case of single-objective identification with genetic algorithm. It can be justified by the strategy of genetic algorithms which consists of determining satisfactory solutions rather than mathematical optimum. Indeed the differences between the values of $F_{\text {err }}^{(2)}$ remain small (inferior to $0.3 \%$ ). One Pareto optimal solution is satisfactory with respect to the definition of the single-objective optimization of the first pressuremeter test (Figure 7), but does not belong to the satisfactory set detected by the single-objective identification with the genetic method. Therefore, the multi-objective formulation allows for the emergence of new satisfactory sets of parameters suitable for the considered problem.

Figure 7 compares the results given by the weighted sum approach using the simplex method and by MOGA-II in terms of Pareto. The solution given by the weighted sum approach is not as good as the three Pareto optimal solutions given by MOGA-II. Indeed, the genetic method provides solutions, whose values of both error functions are inferior to those of the solution given by the weighted sum approach. However, these four sets of parameters are located in the same region of the parameter space as shown by Tables VII and VIII and, therefore, a small difference is to expect in the different calculations of the spread footing test. 


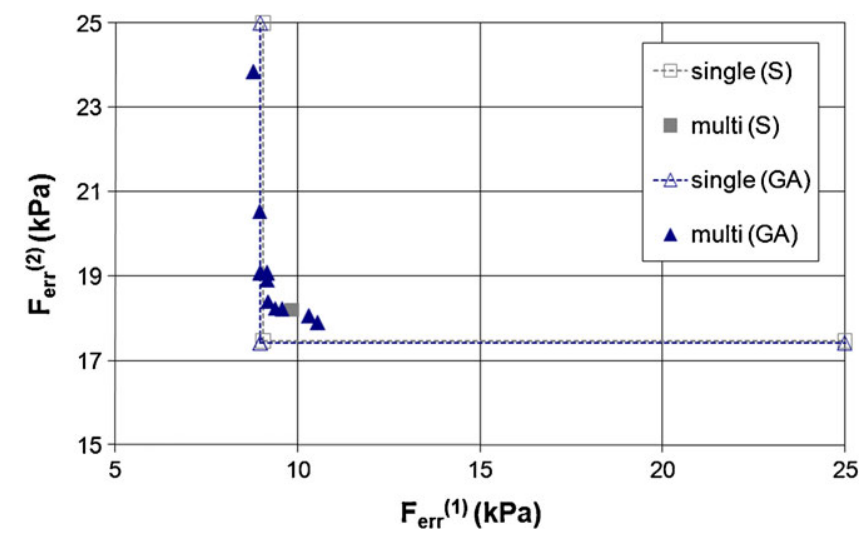

Figure 8. Distribution of Pareto optimal solutions of the multi-objective identification with the simplex (S) and genetic (GA) methods in the criterion space assuming a strain-hardening elasto-plastic model compared with the results of single-objective identification.

Inverse analysis of the first pressuremeter leads systematically to solutions with lower values of the error function. This difference can be related to the different range of pressure during the test.

3.4.2. Identification with the SH-EP model. For the strain-hardening elasto-plastic model, four parameters are optimized: two different values of $a$ : $a_{1}$ (respectively $a_{2}$ ) that correspond to the two different depths $2 \mathrm{~m}$ (respectively $5.9 \mathrm{~m}$ ), one cohesion value $c$ assuming the homogeneity of the soil profile and the value of $E_{\text {ref }}$, assuming the evolution of small strain stiffness given in Equation (10). The same comment about the search of $a_{1}$ and $a_{2}$ can be made as the one previously made for elastic moduli: a unique value of $a$ is not searched for, which takes into account the fact that $a$ is a non-intrinsic parameter. In the same way as in the previous section, optimizations with the simplex and genetic methods were carried out. Figure 8 shows the distribution of Pareto optimal solutions given by the weighted sum approach using the simplex method and by MOGA-II. As in the case of the Mohr-Coulomb model, the solutions given by the genetic method are better in terms of Pareto than the ones given by the weighted sum approach. The difference is less important as in case of the Mohr-Coulomb model, which confirms the comment concerning the topology of the error function.

According to the two multi-objective identifications, we can conclude that MOGA-II is more reliable than the weighted sum approach. It is difficult to compare the computation cost of the two approaches because the weighted sum approach is not carried out completely. Indeed, in order to determine completely the Pareto frontier, multiple sets of weights should have been tested. However, the set $(0.5,0.5)$ corresponding to equal weights applied to both pressuremeter tests gives locally an approximation of the Pareto frontier and allows us to draw some conclusions.

Compared with the single-objective identification, some additional assumptions are made on the soil profile for the multi-objective identification. Indeed, we assume the homogeneity of the soil in terms of $(c, \varphi)$ for the Mohr-Coulomb model and $\left(E_{\text {ref }}, c\right)$ for the strain-hardening elasto-plastic model. We assume also the relation in Equation (10). For both models, and under these assumptions, it is possible to find parameter sets whose values of error functions are close to the satisfactory criteria defined in the single-objective identifications (Figures 7 and 8). Therefore, the multi-objective optimization enables us to verify the consistency of the assumptions made by finding satisfactory compromises. Let us note that, under these assumptions, a different suction effect in the two depths, as mentioned in Section 3.3.2, cannot be taken into account.

The same comment about the two constitutive models as in the single-objective identification can be made in the multi-objective identification. The simplex method encounters the same difficulties for detecting a global minimum, especially under the assumption of the Mohr-Coulomb model. 


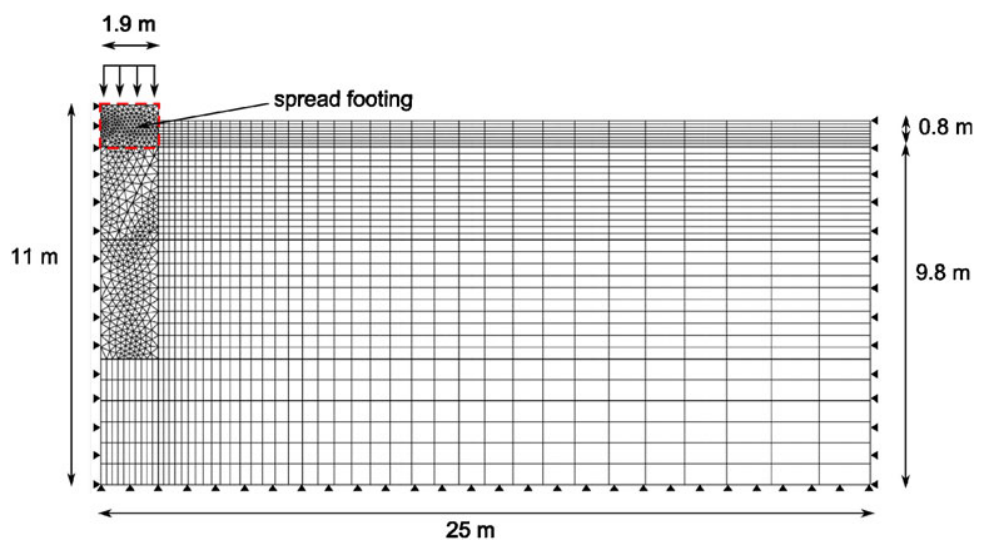

Figure 9. Finite element model of the spread footing test.

However, this tendency is based only on the value of the error functions and on the values of the parameters, which can be deceptive. In order to obtain more elements of analysis on the quality of the identification procedure, we decided to simulate the spread footing test, using the different parameter sets obtained by the previous study.

\subsection{Predictions of spread footing settlement}

The sets of parameters obtained by considering a single- or a multi-objective problem and assuming a Mohr-Coulomb behavior or a strain-hardening elasto-plastic behavior are now used to perform numerical simulations of the spread footing. Since the spread footings are square, a 3D-model should be required. However, a prior study has shown that, for equivalent meshes, the difference of calculated settlements between the 2D- and the 3D-model is small compared with the difference between the computational costs. Therefore, an axisymmetric model is used (see Figure 9), for which the surface of the circular section is equal to the surface of the rectangular one. 6-node triangular elements and 8-node rectangular elements are used. The model contains 6121 nodes and 2278 quadratic elements. Two soil layers are considered in order to carry out simulations with parameters obtained for single- and multi-objective identifications. Therefore, in the case of simulations with parameters obtained from single-objective identifications, both layers are characterized by two different sets of parameters and, in the case of simulations with parameters obtained from multi-objective identifications, two parameters are identical for both layers and one is different. A linear elastic model is used for the concrete slab $(E=11 \mathrm{GPa})$. The loading is force-controlled. Inverse analyses were performed from pressuremeter tests. The soil stiffness in the horizontal direction was, therefore, determined. We assume that the stiffness in the vertical direction is mainly mobilized for the footing test and we retain for the two constitutive models an elastic modulus 1.5 times higher than the one identified, which corresponds to a currently observed ratio between vertical and horizontal stiffness.

Figure 10 compares the experimental and numerical settlement curves resulting from different simulations. A crucial difference exists between the simulations with the Mohr-Coulomb model and the SH-EP model: the latter gives numerical simulations which agree better with the curvature of the experimental settlement curve. The difference can be explained by the hardening function, defined in the SH-EP model, which gives a better representation of the non-linear soil behavior before failure. In this model, the elastic modulus describes the real elastic behavior, whereas in the Mohr-Coulomb model, a secant elastic modulus is defined to reproduce the complete behavior before failure. The difference on soil stiffness was not noticeable in the inverse analysis from pressuremeter tests partly because of the lack of information about the beginning of the experimental curves. Under this condition, we may argue that the use of pressuremeter tests seems to be not suitable for such prediction. However, further studies under different conditions have to be carried out in order to confirm this comment. 


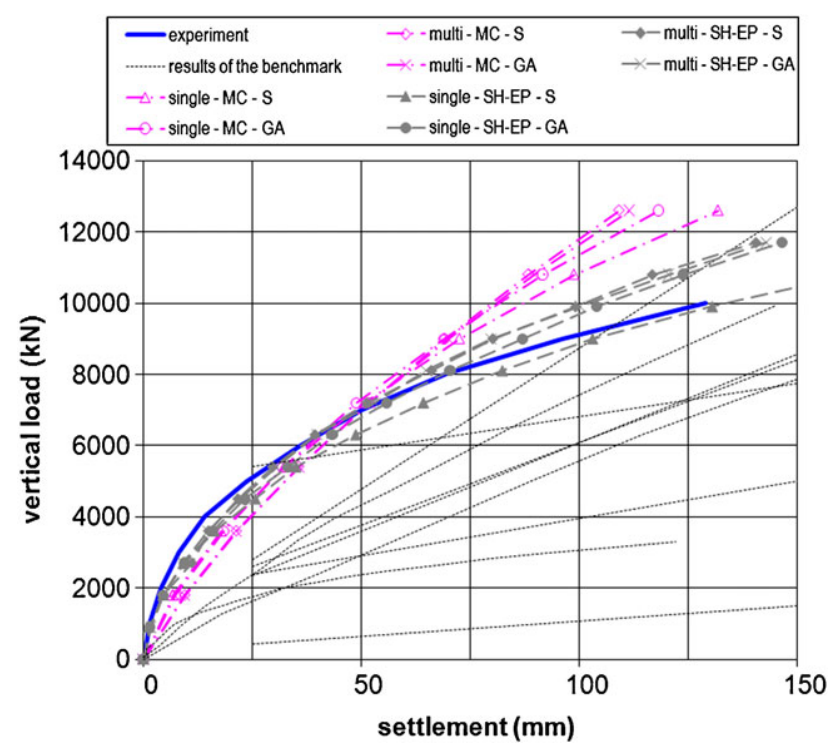

Figure 10. Comparison of experimental spread footing settlements and numerical predictions assuming two constitutive models (Mohr-Coulomb model (MC) and a strain-hardening elasto-plastic model (SH-EP)) resulting from single- and multi-objective identifications with the simplex (S) and genetic (GA) methods.

Compared with most contributors to the benchmark, the predictions for both models comply better with the experimental observations. Note that the results of some participants are given only for 25 and for $150 \mathrm{~mm}$ according to the benchmark requirements. It is obvious that the conditions between this study and the predictions given by the participants of the benchmark are different since the benchmark results were already known. However, the predictions given in Figure 10 result from an objective process. Indeed, the only assumption made in this study, whose consequences are important, concerns the size of the elastic domain $r_{\mathrm{el}}$ and consists of assuming a certain level of overconsolidation. In fact if the beginning of the pressuremeter curves had been known, we would have been able to better determine this parameter. It is thus worth noting the importance of the quality of the identification tests. Considering the predictions of some participants, constitutive parameters used in their studies may have not been properly determined, which can be due to a non-efficient identification technique.

Considering both models separately, the difference between the numerical simulations remains in a limited range. However, for the Mohr-Coulomb model (respectively, the SH-EP model), the simulations resulting from the single-objective identification lead to 5\% (respectively, 12\%) load difference at a $125-\mathrm{mm}$ settlement. This difference was expectable, considering the differences noticed between the parameter values, but was not expectable considering the numerical pressuremeter curves. Indeed, since the stress paths of the identification test, on the one hand, and of the design simulation, on the other hand, are generally different, there is no reason to expect that sets of parameters, which lead to quasi-identical numerical curves for the simulation of the test during inverse analysis, lead also to quasi-identical numerical curves for the design simulation. This remark shows the importance of the choice of the identification test.

For both models, the simulations resulting from multi-objective identifications are close to each other. Therefore, the consistency of the parameter values along the soil profile, guaranteed by the assumptions made on the soil profile, leads for both optimization methods to equivalent sets of parameters in terms of inverse analysis and design calculation. This comment shows the interest of a multi-objective identification by partly overcoming the problem related to the choice of the identification tests. The difference between simulations resulting from the simplex and genetic methods is not significant enough to be commented.

Compared with the simulations resulting from single-objective identifications, the simulations resulting from multi-objective identifications show globally an initially stiffer behavior. 
This difference can be explained by the assumption of homogeneity of the soil profile made for the multi-objective calculation. In the case of the Mohr-Coulomb model, the constant friction angle along the soil profile implies an overestimated friction angle at $2 \mathrm{~m}$ depth which is balanced by a smaller elastic modulus. Therefore, the simulations resulting from multi-objective identifications are less stiff at the beginning of the simulation than the simulations resulting from single-objective identifications, but are stiffer at the end of the simulation. A similar explanation can be proposed in the case of the SH-EP model.

Compared with the experimental spread footing curve, the numerical simulation with the SH-EP model and the set of parameters resulting from the single-objective optimization with the simplex method provides the best fitting. Moreover, the other numerical simulations of the spread footing curve assuming this model seem to be consistent with each other in spite of the small differences discussed previously. The set of parameters, especially the value of the hardening parameter, resulting from the single-objective optimization with the simplex method, is different from the others. However, according to the results provided by the genetic method, this set of parameters is a secondary minimum. This fact implies two observations. First, in terms of inverse analysis, the mathematical best set of parameters is not necessarily the physical best set of parameters considering the noisy error functions due to measurement errors and the imperfect reproduction of the reality by constitutive models. Second, the error made in the parameter identification (i.e. detection of a secondary minimum instead of a global one) might be balanced by the imperfection of the numerical simulation of the spread footing. Indeed, shear bands of high intensity, which appear progressively during the test, cannot be modeled with good accuracy due to the mesh dependency and, therefore, the soil behavior appears more resistant than it is in reality. Parameter identification is thus directly related to design simulation. It is worth noting that the genetic method determined satisfactory sets of parameters close to the best set of parameters obtained with the simplex method. Therefore, if the user performs an inverse analysis with genetic algorithms, he or she can select different satisfactory sets of parameters, whose values are different enough to expect a difference in the design simulation, and carry out the design simulation for each selected set of parameters. The difference between the numerical simulations provides the way to quantify the indecision related to the identification procedure and to deal with the errors due to the imperfection of the numerical simulation.

An inverse analysis performed by using the spread footing test as a reference test could not be carried out due to the implied computational cost.

\section{CONCLUSIONS}

Generally, the usual parameter identification techniques based on inverse analysis remain unsatisfactory, because they do not take into account the non-uniqueness of the inverse analysis problems. This difficulty can be overcome by determining a set of satisfactory solutions and the use of several tests. Along this way, we proposed to compare two kinds of optimization methods. Inverse analysis was carried out from two pressuremeter tests for a subsequent prediction of a spread footing settlement. In order to study the efficiency of optimization methods in two different conditions, two constitutive models were successively examined.

The inverse analysis is considered first as a single-objective problem, so that prior conclusions can be drawn. The computational cost with genetic method is higher, but this method provides more reliable results and more information concerning the parameter values, which can be processed by the user to find suitable parameters. The advantage related to the reliability increases when all the constitutive parameters sensitive to the considered problem have to be identified without any strong restriction on the search space, as shown by the comparison between the results given by inverse analysis for the two models. Therefore, this method seems to become very interesting when the number of parameter to identify becomes bigger.

One of the objectives of this study was to test the capability of the genetic method in the case of the simultaneous identification of several parameters. The inverse analysis was then carried out 
by considering simultaneously two pressuremeter tests. The weighted sum approach appears to be not as reliable as the direct determination of the Pareto frontier by the genetic method, especially in the case of the Mohr-Coulomb model because of the topology of the error function. If enough points of the Pareto frontier are determined, the user can choose the most suitable compromise. If simplified assumptions (for example, the homogeneity of the soil profile) can be made, the multi-objective identification gives the possibility to the user to verify the consistence of these assumptions by finding satisfactory compromises.

The simulation of the spread footing test raises two main difficulties in the identification process which the user must consider. First, inverse analysis is usually applied on experimental tests whose stress path is different from the one concerning the design simulation. Therefore, sets of parameters which lead to quasi-identical numerical curves for the test examined in the inverse analysis do not lead systematically to quasi-identical numerical curves for the design simulation. Moreover, this difficulty increases if only a part of the experimental curve of the test involved in inverse analysis is known as shown by the comparison of the two models. The indecision can be reduced by a multi-objective identification, which provides close numerical simulations for both optimization methods. Second, the apparently successful predictions provided by the singleobjective identification with the simplex method lead us to two comments. Secondary minima can provide better design predictions than global minima considering the errors due to experimental measurement and the imperfect reproduction of the reality by the constitutive models. The error made by selecting a secondary minimum can also be balanced by errors in the design simulation.

Considering the errors or indecisions accumulated due to experimental measurement, the imperfect reproduction of the reality by constitutive models and the nature of identification test or the numerical method for simulation, it is difficult to define a systematic methodology to identify constitutive parameters. However, the genetic method provides a quasi-exhaustive set of satisfactory parameters which can be used to estimate the uncertainty related to the identification procedure.

\section{ACKNOWLEDGEMENTS}

The authors thank J. L. Briaud (Texas A\&M University) for making available information about the spread footing benchmark.

\section{REFERENCES}

1. Duncan JM, Chang CY. Non linear analysis of stress and strain in soils. Journal of Soil Mechanics and Foundations Division (ASCE) 1970; 96:1629-1653.

2. Lade PV. Double hardening constitutive model for soils, parameter determination and predictions for two sands. In Constitutive Equations for Granular Noncohesive Soils, Saada A Bianchini G (eds). Balkema: Rotterdam, 1988; 367-382.

3. Kolymbas D. Computed-aided design of constitutive laws. International Journal for Numerical and Analysis Methods in Geomechanics 1991; 15:593-604.

4. Mestat P, Arafati N. Modélisation des sables avec la loi de Nova: détermination des paramètres et influence sur les simulations. LPC Bulletin 2000; 225:21-40.

5. Gioda G. Some remarks on back analysis and characterization problems in geomechanics. In Fifth International Conference on Numerical Methods in Geomechanics, Nagoya, Japan, Kawamoto T, Ichikawa Y (eds). Balkema: Rotterdam, 1985; 47-61.

6. Arai K, Ohta H, Kojima K. Estimation of soil parameters based on monitored movement of subsoil under consolidation. Soils and Foundations 1984; 24(4):95-108.

7. Pal S, Wathugala WG, Kundu S. Calibration of a constitutive model using genetic algorithms. Computers and Geotechnics 1996; 19:325-348.

8. Levasseur S, Malécot Y, Boulon M, Flavigny E. Soil parameter identification using a genetic algorithm. International Journal for Numerical and Analytical Methods in Geomechanics 2008; 32:189-213.

9. Levasseur S, Malécot Y, Boulon M, Flavigny E. Statistical inverse analysis based on genetic algorithm and principal component analysis: method and developments using synthetic data. International Journal for Numerical and Analytical Methods in Geomechanics 2009; 33:1485-1511.

10. Levasseur S, Malécot Y, Boulon M, Flavigny E. Statistical inverse analysis based on genetic algorithm and principal component analysis: applications to excavation problems and pressuremeter tests. International Journal for Numerical and Analytical Methods in Geomechanics 2010; 34:471-491. 
11. Briaud JL, Gibbens RM. Predicted and measured behavior of five spread footings on sand. Geotechnical Special Publication 41, ASCE, New-York, 1994.

12. Tarantola A. Inverse Problem Theory and Methods for Model Parameter Estimation. SIAM: Philadelphia, 2005.

13. Deb K. Multi-Objective Optimization using Evolutionary Algorithms. Wiley: New York, 2001.

14. Pareto V. Cours d'Economie Politique. Rouge: Lausanne, 1897.

15. Calvello M, Finno RJ. Selecting parameters to optimize in model calibration by inverse analysis. Computers and Geotechnics 2004; 31:411-425.

16. Finno RJ, Calvello M. Supported excavations: the observational method and inverse modeling. Journal of Geotechnical and Geoenvironmental Engineering (ASCE) 2005; 131(7):826-836.

17. Lecampion B, Constantinescu A, Nguyen Minh D. Parameter identification for lined tunnels in viscoplastic medium. International Journal for Numerical and Analytical Methods in Geomechanics 2002; 26:1191-1211.

18. Zentar R, Hicher PY, Moulin G. Identification of soil parameters by inverse analysis. Computers and Geotechnics 2001; 28:129-144.

19. Dano C, Hicher PY, Rangeard D, Marchina P. Interpretation of dilatometer tests in a heavy oil reservoir. International Journal for Numerical and Analytical Methods in Geomechanics 2006; 31:1197-1215.

20. Rangeard D, Hicher PY, Zentar R. Determining soil permeability from pressuremeter tests. International Journal for Numerical and Analytical Methods in Geomechanics 2003; 27:1-24.

21. Nelder JA, Mead R. A simplex method for function minimization. Computer Journal 1965; 7:308-313.

22. Ledesma A, Gens A, Alonso EE. Estimation of parameters in geotechnical backanalysis-I. Maximum likehood Approach. Computers and Geotechnics 1996; 18(1):1-27.

23. Gens A, Ledesma A, Alonso EE. Estimation of parameters in geotechnical backanalysis-II. Application to a tunnel excavation problem. Computers and Geotechnics 1996; 18(1):29-46.

24. Hicher PY, Rahma A. Micro-macro correlations for granular media. Application to the modelling of sands. European Journal of Mechanics, A/Solids 1994; 13(6):763-781.

25. Obrzud RF, Vulliet L, Truty A. Optimization framework for calibration of constitutive models enhanced by neural networks. International Journal for Numerical and Analytical Methods in Geomechanics 2009; 33:71-94.

26. Mertens J, Stenger R, Barkle GF. Multiobjective inverse modeling for soil parameter estimation and model verification. Vadose Zone Journal 2006; 5:917-933.

27. Holland JH. Adaptation in Natural and Artificial Systems. University of Michigan Press: Ann Arbor, MI, 1975.

28. Goldberg DE. Genetic Algorithms in Search, Optimization and Machine Learning. Adisson-Wesley: Reading, MA, 1989.

29. Simpson AR, Dandy GC, Murphy LJ. Genetic algorithms compared to other techniques for pipe optimization. Journal of Water Resources Planning and Management 1994; 120(4):423-443.

30. Samarajiva P, Macari EJ, Wathugala W. Genetic algorithms for the calibration of constitutive models for soils. International Journal of Geomechanics 2005; 5(3):206-217.

31. Poles S, Rigoni E, Robic T. MOGA-II Performance on noisy optimization problems. In International Conference on Bioinspired Optimization Methods and their Applications-BIOMA 2004, Ljubljana, Slovenia, Filipic B, Silc J (eds). Jozef Stefan Institute: Ljubljana, 2004; 51-62.

32. Poloni C, Pediroda V. GA coupled with computationally expensive simulations: tools to improve efficiency. In Genetic Algorithms and Evolution Strategies in Engineering and Computer Science. Recent Advances and Industrial Applications, Quagliarella D, Périaux J, Poloni C, Winter G (eds). Wiley: West Sussex, England, 1997; 267-288.

33. Rechea C, Levasseur S, Finno RJ. Inverse analysis techniques for parameter identification in simulation of excavation support systems. Computers and Geotechnics 2008; 35(3):331-345.

34. Cambou B, Bahar R. Utilisation de l'essai pressiométrique pour l'identification de paramètres intrinsèques du comportement du sol. Revue Française de Géotechnique 1993; 63:39-50.

35. Bahar R. Analyse numérique de l'essai pressiométrique: application à l'identification de paramètres de comportement des sols. Ph.D. Thesis, Ecole Centrale Lyon, 1992.

36. Dano C, Hicher PY. A constitutive model for uncemented and grouted sands. In Fifth European Conference on Numerical Methods in Geotechnical Engineering-NUMGE 2002, Paris, France, Mestat P (eds). Presses de l'ENPC/LCPC: Paris, 2002; 57-62.

37. Bardet JP. Lode dependences for isotropic pressure-sensitive elastoplastic materials. Journal of Applied Mechanics 1990; 57:498-506.

38. Biarez J, Hicher PY. Elementary Mechanics of Soil Behaviour. Balkema: Rotterdam, 1994.

39. Hicher PY. Elastic properties of soils. Journal of Geotechnical Engineering 1996; 122(8):641-648.

40. ModeFrontier 4, User Manual, Esteco, Trieste, Italy, 2009.

41. Sobol IM. Distribution of points in a cube and approximate evaluation of integrals. USSR Computational Mathematics and Mathematical Physics 1967; 7:86-112.

42. Renders JM. Algorithmes Génétiques et Réseaux de Neurones. Hermès: Paris, 1995. 